\title{
Electron-phonon coupling and intervalley splitting determine the linewidth of single-electron transport through $\mathrm{PbSe}$ nanocrystals
}

\author{
K. Overgaag, ${ }^{1}$ D. Vanmaekelbergh, ${ }^{1}$ P. Liljeroth, ${ }^{1, a)}$ G. Mahieu, ${ }^{2}$ B. Grandidier, ${ }^{2, b)}$ \\ C. Delerue, ${ }^{2}$ and G. Allan ${ }^{2}$ \\ ${ }^{1}$ Condensed Matter and Interfaces, Debye Institute for Nanomaterials Science, Utrecht University, \\ P.O. Box 80000, 3508 TA Utrecht, The Netherlands \\ ${ }^{2}$ Département ISEN, Institut d'Electronique de Microélectronique et de Nanotechnologie, IEMN, \\ (CNRS, UMR 8520), 41 bd Vauban, 59046 Lille Cedex, France
}

(Received 2 April 2009; accepted 17 November 2009; published online 9 December 2009)

\begin{abstract}
The linewidth of the resonances in the single-electron tunneling spectra has been investigated for $\mathrm{PbSe}$ semiconductor nanocrystals (NCs) with scanning tunneling spectroscopy at low temperature. The linewidth of the resonances corresponding to tunneling through the first conduction and valence levels is found to increase with decreasing size of the NCs. Based on theoretical calculations, this broadening is mainly induced by the coupling between the tunneling electrons and the longitudinal optical phonon mode of the $\mathrm{NC}$, and by the splitting of the degenerate electronic levels between the different L-valleys in the Brillouin zone. For the smallest sizes, it is shown that the intervalley splitting is the major source of broadening. (C) 2009 American Institute of Physics.
\end{abstract}

[doi:10.1063/1.3272952]

Colloidal semiconductor nanocrystals (NCs) are an experimental realization of the textbook "particle in a box" problem in quantum mechanics. As a result of quantum confinement, their energy levels are discrete with atomlike symmetries (S, P, etc.) and can be tuned by the NC size. The tunability of the optoelectronic properties of these semiconductor NCs makes them attractive for use in a variety of light emitting technologies. ${ }^{1-3}$ In contrast to such optical applications, prototypes of electronic devices made up of NCs are quite limited. ${ }^{4}$ Increasing the number of applications will strongly depend on our ability to understand the physical mechanisms involved in the charge transport through single NCs.

The electronic structure and single-electron charging of colloidal semiconductor NCs can be studied in the doublebarrier tunnel junction geometry by scanning tunneling spectroscopy (STS) at low temperatures. The single-electron tunneling spectrum, i.e., $d I / d V_{\text {bias }}$ versus $V_{\text {bias }}$, consists of a number of peaks (resonances) that directly reveal the discrete energy levels of the $\mathrm{NC}^{5-9}$ More recently, it was shown that also the linewidth of the resonances can be used to obtain new insight into the physical parameters governing the transport, such as the strength of the electron-phonon coupling. ${ }^{10}$

Here, we investigate the linewidth of the resonances in tunneling spectra of PbSe NCs. As the size of the $\mathrm{NC}$ is reduced, we observe significant broadening of the resonances. Based on theoretical estimates, this broadening is too strong to be caused solely by the coupling between the tunneling electrons and longitudinal optical (LO) phonons. In contrast to the archetypical II-VI semiconductor NCs, $\mathrm{PbSe} \mathrm{NCs}$ have a rocksalt-structure crystal structure with a narrow band gap at the L point of the Brillouin zone. Due to

\footnotetext{
a)Electronic mail: p.liljeroth@uu.nl.

${ }^{b)}$ Electronic mail: bruno.grandidier@isen.iemn.univ-lille1.fr.
}

quantum confinement, the intervalley coupling splits the lowest L states and for sufficiently small NCs, the magnitude of the splitting may become of the order of the measured linewidth corresponding to tens of meV. ${ }^{11-15}$ Comparison between experiment and tight-binding calculations shows that the intervalley splitting is the dominant contribution to the linewidth for PbSe NCs smaller than $5 \mathrm{~nm}$.

Spherical PbSe NC were synthesized according to the literature methods ${ }^{16,17}$ and recapped with hexylamine. From the controlled growth of these colloids, four different sets of NCs were prepared, having average diameters of 4.1, 5.3, 7.1 , and $9.8 \mathrm{~nm}$, as determined by transmission electron microscopy (TEM). A flame-annealed $\mathrm{Au}(111)$ substrate with a self-assembled hexanedithiol monolayer was immersed in a dispersion of colloidal $\mathrm{PbSe} \mathrm{NCs}$ in chloroform leading to stable adsorption of individual NCs [Fig. 1(a)]. The samples were then loaded in an ultrahigh vacuum system containing a low-temperature scanning tunneling microscope (LT STM, Omicron Nanotechnology) to be annealed at $150{ }^{\circ} \mathrm{C}$ for several hours and then transferred into the microscope to perform STS experiments on individual NCs. The tunneling differential conductance $d I / d V_{\text {bias }}$ was obtained by measuring the dynamic differential conductance using a lock-in amplifier $\left(V_{\bmod }=7-10 \mathrm{mV}, f_{\bmod }=0.5-1.0 \mathrm{kHz}\right)$ at a temperature of $4.8 \mathrm{~K}$. Typically a large number of curves were acquired above a single NC under identical feedback conditions. The measured curves were reproducible, although discrete shifts of the spectra along the bias axis were observed in some cases. This is most likely related to a charge fluctuation in the surrounding of the NC. ${ }^{10}$ Because the variation of the electron energy in the $\mathrm{NC}$ becomes smaller and smaller as the external charge resides further away from the $\mathrm{NC}$, we minimize the influence of the charge fluctuations on the level broadening by averaging only curves, where the main peaks are shifted by less than 

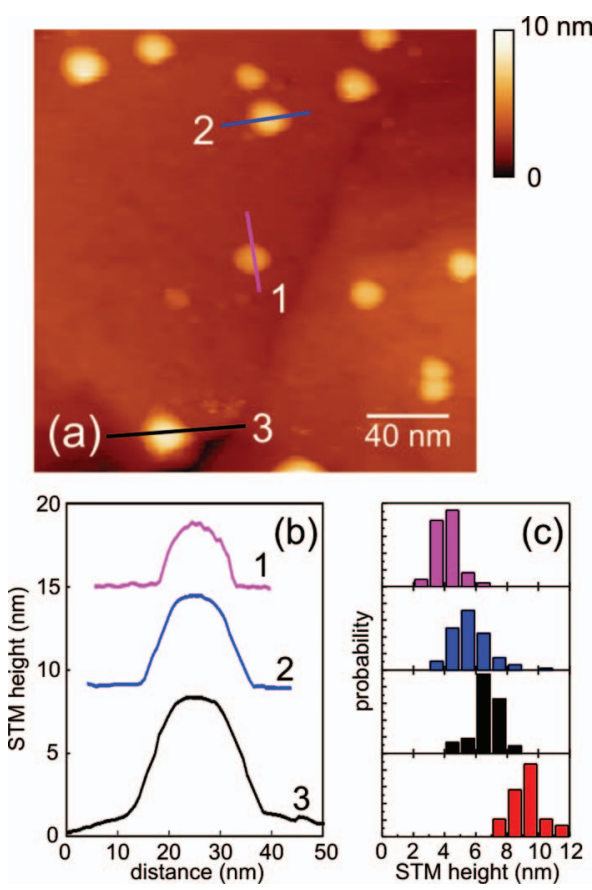

FIG. 1. (a) STM topographic image of PbSe NCs chemically linked by a hexanedithiol self-assembled monolayer to a $\mathrm{Au}(111)$ surface. The image was acquired with a sample voltage $V_{\text {bias }}=2.5 \mathrm{~V}$ and a current set-point $I_{\mathrm{t}}=20 \mathrm{pA}$. (b) Three different height profiles measured in the topography image to determine the STM height of the NCs. (c) STM height histograms on four different samples with PbSe NCs with average core diameters of 4.1, 5.3, 7.1, and $9.8 \mathrm{~nm}$ (measured by TEM).

+/-4 $\mathrm{mV}$ between the curves. As a result, charge fluctuations do not widen the peak linewidth by more than $8 \mathrm{mV}$ and averaging at least ten individual curves per tunneling spectrum usually allows to substantially increase the signalto-noise ratio.

Figure 1 shows a STM image of the Au(111) surface with well-separated individual PbSe NCs. This particular sample was prepared using a mixture of different sizes of PbSe NCs. Although the tip convolution effect generally makes the lateral particle size bigger than the expected diameter, the measured STM height closely reflects the NC core diameter, when the bias voltage is set outside the NC zero-conductivity gap. ${ }^{8}$ The height profiles yield values of $3.9,5.6$, and $6.9 \mathrm{~nm}$ corresponding to the average NC diameters of 4.0, 5.3, and $7.1 \mathrm{~nm}$ measured by TEM. The general correspondence between the STM height and TEM diameter is illustrated in Fig. 1(c), where we show height histograms from several STM images, again confirming one-to-one correspondence between the size measurement by STM and TEM.

After the measurement of the NC size, we can stabilize the STM tip on top the NC for STS measurements. Figure 2(a) gives five examples of tunneling spectra measured for NCs with sizes ranging from 3.5 to $10.2 \mathrm{~nm}$. The tunneling spectra consist of a zero-conductivity gap surrounded by sequences of peaks at positive and negative sample voltages. These conductance peaks correspond to the conduction (electron levels) and valence (hole levels) orbitals of the NCs, respectively. Both the zero-conductivity gap as well as the level separations increase with decreasing NC diameter, con-
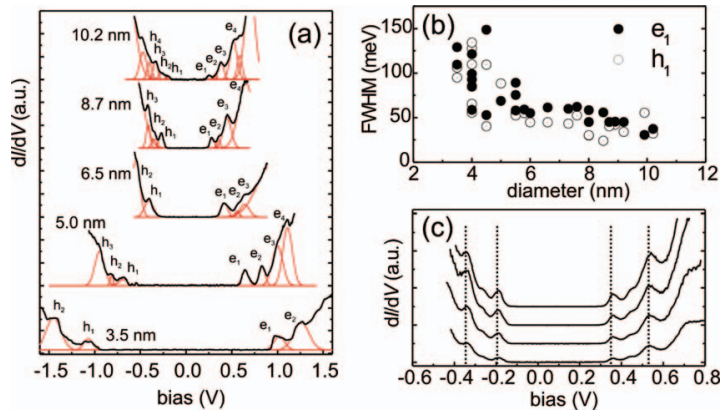

FIG. 2. Spectroscopy of PbSe NCs chemically linked to Au(111) with hexanedithiol. (a) Effect of the NC diameter (determined by STM height profiles). Parameters typically used are $I_{\mathrm{t}}=50$ to $400 \mathrm{pA}$ and $V_{\text {bias }}=0.7$ to $1.6 \mathrm{~V}$. The spectra are fitted with Gaussian functions and the resonances are denoted $h_{1}$ to $h_{4}$ for the valence levels and $e_{1}$ to $e_{4}$ for the conduction levels. (b) Variation of the FWHM of the resonances $e_{1}$ and $h_{1}$ as a function of the NC diameter. (c) Spectroscopy measurements with different tunneling current set-points $I_{\mathrm{t}}$. NC size $=7.7 \mathrm{~nm}, V_{\text {bias }}=0.8 \mathrm{~V}$, $I_{\mathrm{t}}=200,400,550,700 \mathrm{pA}$ from bottom to top.

sistent with a stronger quantum confinement in smaller NCs. While some resonances at higher bias are difficult to resolve depending on the NC size, the first resonances $\mathrm{e}_{1}$ and $\mathrm{h}_{1}$, corresponding to the lowest electron and hole orbitals with $\mathrm{S}$ envelope symmetry, are always clearly seen. We thus focus the analysis of the linewidth of these resonances.

Fitting the data shown in Fig. 2(a) with Gaussians reveals that the full width at half maximum (FWHM) of the $\mathrm{e}_{1}$ and $h_{1}$ resonances increases as the size of the NCs decreases as shown in Fig. 2(b). There is a clear change in the slope of the points: the measured FWHM increases more abruptly for NCs with a diameter smaller than $5 \mathrm{~nm}$. In order to verify that this is not a spurious effect due to a change in the potential distribution in the tip-NC-substrate tunneling junction with different set-point conditions, we first measured set of spectra on the same NCs with different tunneling current set points. Figure 2(c) confirms that the apparent band gap as well as the linewidth of the resonances is independent of the current set point, implying that the small changes in the tip-NC distance caused by the changes in the current set point do not significantly change the potential distribution in the tip-NC-substrate junction. In addition, the number of peaks does not change with increasing current set point; the spectra are measured under shell-tunneling conditions and the resonances directly correspond to single-electron energy levels of the NCs. ${ }^{6,7,9,18}$ A constant linewidth as a function of the current also indicates that the heat is well-dissipated and the local temperature of the NC is not increased due to inelastic tunneling events. ${ }^{10}$

Coupling of the electron to phonon modes in nanostructures is known to be a source of broadening in transport ${ }^{19,20}$ and the contribution of vibronic states in the tunneling current flowing through single atoms, molecules, and NCs have recently been demonstrated. ${ }^{21-23}$ Similarly, polaronic transport is expected in our experiments and the strength of the electron-phonon coupling should increase with the decreasing NC size. If the intrinsic linewidth of the phonon replica is larger than their spacing (given by the phonon energy), we expect a single broadened resonance instead of a series of peaks. To account for this effect, we estimated the coupling 


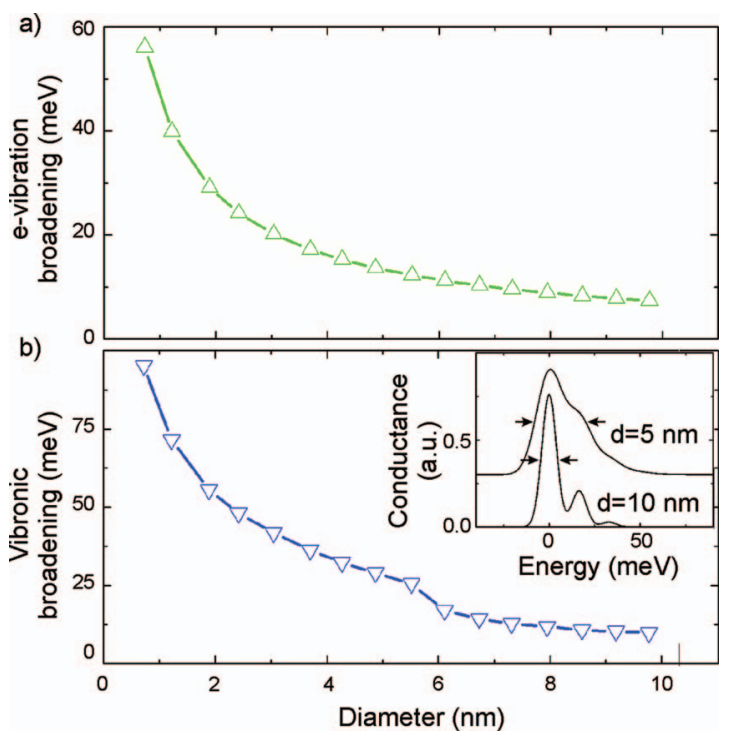

FIG. 3. (a) Theoretically estimated broadening of the differential conductance linewidth due to the polarization of the molecules in response to the electric field induced by an electron tunneling into a S state of a NC. The molecules are modeled as a dielectric layer $[\varepsilon(0)=20$ and $\varepsilon(\infty)=2]$ with a thickness of $2 \mathrm{~nm}$. (b) Theoretically estimated evolution of the differential conductance linewidth, resulting from electrons tunneling into a $\mathrm{S}$ state coupled to LO phonons, as a function of the NC size. The broadening includes the contribution of the polarization of the capping molecules in response to the electric field induced by the electron. Inset: examples of theoretical line shapes for the differential conductance peak for NC diameters $d$ of 5 and $10 \mathrm{~nm}$. The line shape corresponds to a series of Dirac peaks (LO phonon replica) broadened by a Gaussian function. The FWHM of the Gaussian function corresponds to the broadening caused by the electronvibration coupling between the electron and the surrounding molecules. The arrows indicate where the FWHM of the differential conductance linewidth is determined.

between the tunneling electrons and the LO phonon mode in the NC based on the theory described in Ref. 10. In addition to the polar interaction between the electronic charge density and the PbSe phonons, the electric field induced by the injected charge polarizes the organic ligands (capping molecules) surrounding the NCs. In analogy to the calculations described in the Appendix B of Ref. 10, the capping molecules can be considered as a dielectric layer of thickness $2 \mathrm{~nm}$ and of static and dynamical dielectric constants $\varepsilon(0)=20$ and $\varepsilon(\infty)=2$. As shown in Fig. 3(a), the polarization of this surrounding layer also causes an additional broadening of the linewidth, however, to a lesser extent than the coupling between the electron and the NC phonons. As a result, the shape of the differential conductance peak corresponds to a series of Dirac peaks (LO phonon replica) that are convoluted with a Gaussian function. The FWHM of the Gaussian function is given by the broadening induced by the polarization of the organic layer surrounding the $\mathrm{NC}$ and this broadening increases as the size of the NC decreases, as shown in the inset of Fig. 3(b). As a whole, Fig. 3(b) shows that polaronic transport does induce an increase in the resonance linewidths with decreasing NC size. Because both sources of broadening depend roughly as $1 / d$ on the $\mathrm{NC}$ diameter, it is, however, not sufficient to account for the experimentally observed strong increase in the measured FWHM with decreasing NC size.

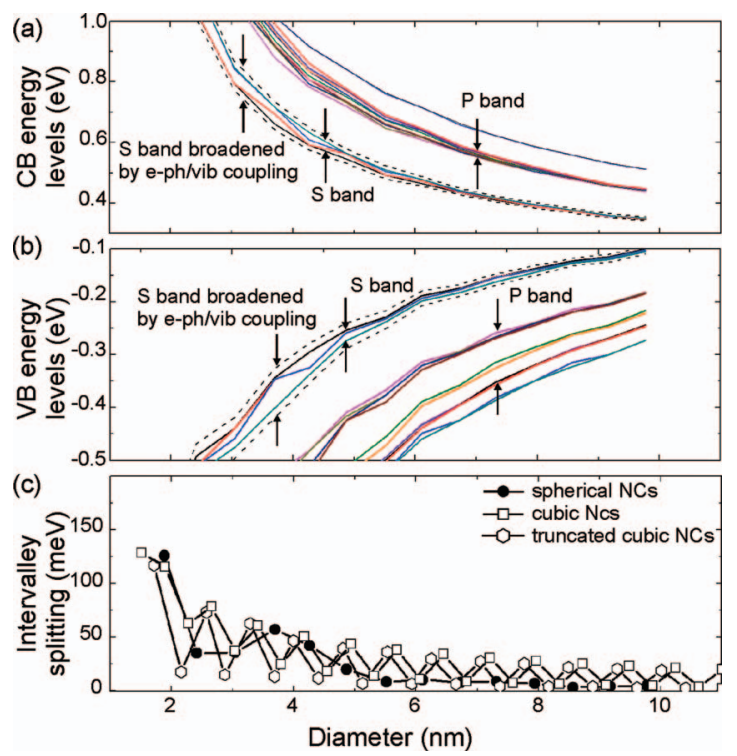

FIG. 4. (a) Energy of the electron levels in the conduction band as a function of the NC size. The lowest levels are grouped in bands of four S-type levels and twelve P-type levels. (b) Energy of the hole levels in the valence band as a function of the NC size. The highest levels are grouped in bands of four S-type levels and twelve P-type levels. In both graphs, the additional broadening caused by the coupling of the electrons with PbSe phonons and vibrations of the capping molecules is marked by dashed lines. The zero in energy corresponds to the top of the valence band for bulk PbSe. (c) Intervalley splitting of the $\mathrm{S}$ levels in the valence band for NCs with a spherical (closed circles), cubic (open squares), and truncated cubic (hexagons) shape.

$\mathrm{PbSe}$ is known to have a unique electronic structure with fourfold degenerate $S$ levels (without the spin). ${ }^{11,14,15}$ In the $\mathrm{NCs}$, the translational symmetry of the bulk crystal is broken and intervalley coupling is expected to cause a substantial splitting of the levels. Using tight binding calculations based on the method described in Ref. 14, we obtained the electronic structure of PbSe NCs as a function of their diameter and shape. The energy of the lowest conduction band states are shown in Fig. 4(a) in the case of spherical NCs. The four lowest states have $S$ envelope wave function symmetry and form the $\mathrm{S}_{\mathrm{e}}$ band. The next twelve states correspond to $\mathrm{P}_{\mathrm{e}}$ states, while the two highest states belong to the $D_{e}$ band (which has in total 20 states, only two of them are shown in Fig. 4). Focusing on the $S$ band, the energy splitting between the highest and lowest level among these four $\mathrm{S}_{\mathrm{e}}$ states yields the intervalley splitting for the $S_{e}$ band. The $S$ hole energy levels behave very similarly to the $\mathrm{S}$ electron levels as can be seen in Fig. 4(b). From Figs. 4(a) and 4(b), it is clear that the splitting of the $S_{e}$ and $S_{h}$ bands increases when the size of the NC decreases. By broadening each $\mathrm{S}$ level by the theoretical vibronic progression obtained from the coupling of electrons to LO phonons and to the vibrations of the capping molecules, we obtain the total theoretical broadening of the differential conductance peaks. This corresponds to the energy separation between the two dashed lines for a given NC size as indicated in Fig. 4.

$\mathrm{PbSe}$ NCs are inherently facetted and their shape deviates from a perfect sphere. To take into account of variations in the shape of the NCs, the electronic structure of cubicshaped and truncated cubic-shaped NCs was also calculated. 


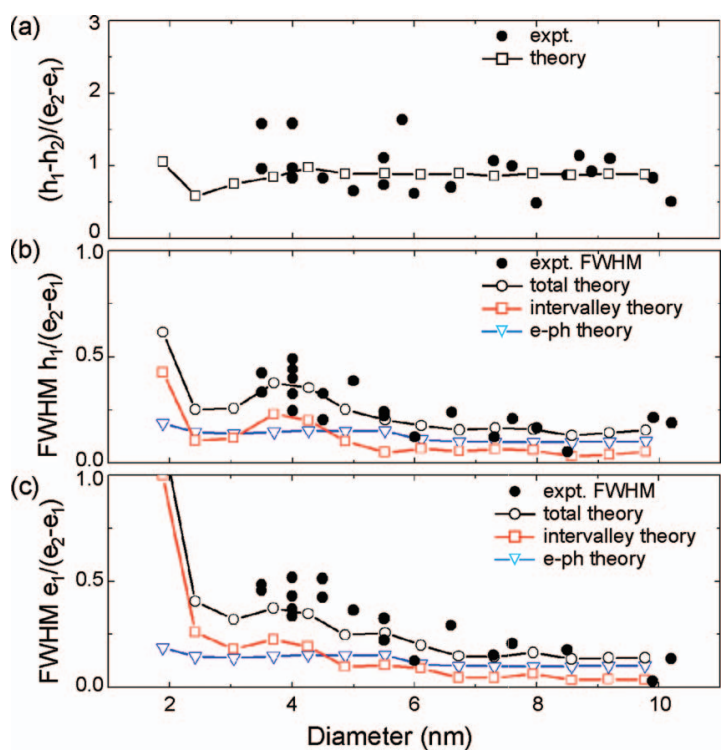

FIG. 5. (a) Measured ratio of the S-P energy level separation between the valence and conduction states (filled circles) compared with tight-binding calculations (open squares). (b) FWHM of the $\mathrm{h}_{1}$ resonance normalized by the $e_{1}-e_{2}$ energy separation as a function of the NC diameter (filled circles). The theoretical results for spherical NCs are shown in open circles, based on the calculated contributions of the intervalley splitting (open squares) and electron-phonon/vibration coupling (downward triangles). (c) FWHM of the $\mathrm{e}_{1}$ resonance normalized by the $\mathrm{e}_{1}-\mathrm{e}_{2}$ energy separation as a function of the NC diameter (filled circles). The theoretical results for spherical NCs are shown in open circles, based on the calculated contribution of the intervalley splitting (open squares) and electron-phonon/vibration coupling (downward triangles). The contribution of the voltage modulation applied to measure the differential conductance has been subtracted in the data related to the experimental FWHM.

From Fig. 4(c), it can be seen that, for NCs with nonspherical symmetries, the intervalley splitting in the valence band increases with distinct sawtooth behavior, which is caused by an odd and even numbers of PbSe planes depending on the $\mathrm{NC}$ size. A similar result is found for the conduction band. Indeed the intervalley coupling can be written as matrix elements of the potential perturbation induced by the facets between two Bloch states at different minima of the conduction or valence band, resulting in strong oscillations of the splitting, depending on the nature of the facets. ${ }^{14,24}$

Due to the crystal structure of $\mathrm{PbSe}$ and nearly equal effective masses of electrons and holes, the valence and conduction bands have a similar level structure, as shown theoretically in Fig. 4. This can be experimentally assessed by comparing the energy separations of the highest levels in the valence band $\left(h_{1}-h_{2}\right)$ with the lowest levels in the conduction band $\left(\mathrm{e}_{2}-\mathrm{e}_{1}\right)$. These energy separations are affected by the potential distribution in the tip-NC-substrate junction. The potential distribution causes the measured spacing between the resonances to increase by a factor of $1 / \eta$, where $\eta$ is the ratio of the potential drop between the STM tip and $\mathrm{NC}$ and the total applied bias $\eta=V_{\text {tip-NC }} / V_{\text {bias. }}$. Since both $\mathrm{h}_{1}-\mathrm{h}_{2}$ and $\mathrm{e}_{1}-\mathrm{e}_{2}$ are affected by $\eta$ in the same way, dividing $\left(\mathrm{h}_{1}-\mathrm{h}_{2}\right)$ by $\left(\mathrm{e}_{2}-\mathrm{e}_{1}\right)$ cancels out the effect of the potential distribution in the tip-NC-substrate double barrier tunnel junction. As shown in Fig. 5(a), this ratio is close to unity for all measured NC diameters and agrees well with the theoret- ical predictions. Such a normalization procedure thus ensures that the experimental spectral features can be compared with the theoretical band structure. ${ }^{10}$

The width of the tunneling resonances is affected in exactly the same manner: the peak broadening depends on $\eta$, in addition to the discussed broadening mechanisms (electronphonon and electron-vibration coupling, intervalley splitting). In Figs. 5(b) and 5(c), we plot the variations of the normalized linewidth for the resonances $h_{1}$ and $e_{1}$. Again, dividing the linewidth by the energy separation $\left(e_{2}-e_{1}\right)$ yields a quantity that is independent of the potential distribution in the double-barrier tunnel junction and allows direct comparison between theory and experiment. The measured normalized linewidth slightly increases with decreasing NC diameter. This trend cannot be explained by only considering broadening due to the electron-phonon and electronvibration couplings, which lead to a slightly decreasing normalized linewidth with decreasing NC diameter. However, once we include the broadening due to intervalley splitting, the theoretical predictions closely match the measured values [Figs. 5(b) and 5(c)]. Intervalley splitting is negligible for the biggest NCs and the linewidth is mainly caused by the electron-phonon and electron-vibration coupling. On the other hand, for $\mathrm{NC}$ diameters below $5 \mathrm{~nm}$, the intervalley splitting becomes the main source of broadening. Theoretical calculations predict strong oscillations in the intervalley splitting depending on the $\mathrm{NC}$ shape and the number of PbSe atomic planes separating the two opposite facets; these oscillations can be seen in the experimental results as increased scatter of the FWHM for small NCs.

In conclusion, we have studied the linewidth of the resonances in $\mathrm{PbSe} \mathrm{NCs}$ corresponding to the electron and hole levels with $\mathrm{S}$ envelope wave function symmetry by singleelectron transport: the smaller the NCs, the broader the resonances are. This broadening is mainly caused by two different physical mechanisms: electron-phonon coupling and intervalley coupling between the different $\mathrm{L}$ points in the Brillouin zone. Comparison between theory and experiment indicates that the latter effect dominates for NCs smaller than $5 \mathrm{~nm}$ in diameter.

The authors wish to acknowledge financial support from NWO/Chemical Sciences (Vidi-Grant No. 700.56.423, P.L.), the European Community ("HERODOT" Project-Grant No. PITN-GA-2008-214954) and Nord Pas de Calais region. We are thankful to Rolf Koole, Arjan Houtepen, and Stephen G. Hickey for the NC samples used in this study.

${ }^{1}$ M. Bruchez, Jr., M. Moronne, P. Gin, S. Weiss, and A. P. Alivisatos, Science 281, 2013 (1998).

${ }^{2}$ S. Coe, W.-K. Woo, M. Bawendi, and V. Bulovic, Nature (London) 420, 800 (2002).

${ }^{3}$ M. Achermann, M. A. Petruska, S. Kos, D. L. Smith, D. D. Koleske, and V. I. Klimov, Nature (London) 429, 642 (2004).

${ }^{4}$ D. V. Talapin and C. B. Murray, Science 310, 86 (2005).

${ }^{5}$ U. Banin, Y. W. Cao, D. Katz, and O. Millo, Nature (London) 400, 542 (1999).

${ }^{6}$ E. P. A. M. Bakkers, Z. Hens, A. Zunger, A. Franceschetti, L. P. Kouwenhoven, L. Gurevich, and D. Vanmaekelbergh, Nano Lett. 1, 551 (2001).

${ }^{7}$ L. Jdira, P. Liljeroth, E. Stoffels, D. Vanmaekelbergh, and S. Speller, Phys. Rev. B 73, 115305 (2006). 
${ }^{8}$ P. Liljeroth, P. A. Zeijlmans van Emmichoven, S. G. Hickey, H. Weller, B. Grandidier, G. Allan, and D. Vanmaekelbergh, Phys. Rev. Lett. 95, 086801 (2005).

${ }^{9}$ U. Banin and O. Millo, Annu. Rev. Phys. Chem. 54, 465 (2003).

${ }^{10}$ L. Jdira, K. Overgaag, R. Stiufiuc, B. Grandidier, C. Delerue, S. Speller, and D. Vanmaekelbergh, Phys. Rev. B 77, 205308 (2008).

${ }^{11}$ I. Kang and F. W. Wise, J. Opt. Soc. Am. B 14, 1632 (1997).

${ }^{12}$ A. D. Andreev and A. A. Lipovskii, Phys. Rev. B 59, 15402 (1999).

${ }^{13}$ F. W. Wise, Acc. Chem. Res. 33, 773 (2000).

${ }^{14}$ G. Allan and C. Delerue, Phys. Rev. B 70, 245321 (2004).

${ }^{15}$ J. M. An, A. Franceschetti, S. V. Dudiy, and A. Zunger, Nano Lett. 6, 2728 (2006).

${ }^{16}$ C. B. Murray, S. Sen, W. Gaschler, H. Doyle, T. A. Betley, and C. R. Kagan, IBM J. Res. Dev. 45, 47 (2001).

${ }^{17}$ A. J. Houtepen, R. Koole, D. Vanmaekelbergh, J. Meeldijk, and S. G.
Hickey, J. Am. Chem. Soc. 128, 6792 (2006).

${ }^{18}$ P. Liljeroth, L. Jdira, K. Overgaag, B. Grandidier, S. Speller, and D. Vanmaekelbergh, Phys. Chem. Chem. Phys. 8, 3845 (2006).

${ }^{19}$ M. C. Klein, F. Hache, D. Ricard, and C. Flytzanis, Phys. Rev. B 42, 11123 (1990).

${ }^{20}$ N. S. Wingreen, K. W. Jacobsen, and J. W. Wilkins, Phys. Rev. B 40, 11834 (1989).

${ }^{21}$ M. Berthe, A. Urbieta, L. Perdigão, B. Grandidier, D. Deresmes, C. Delerue, D. Stiévenard, R. Rurali, N. Lorente, L. Magaud, and P. Ordejón, Phys. Rev. Lett. 97, 206801 (2006).

${ }^{22}$ S. W. Wu, G. V. Nazin, X. Chen, X. H. Qiu, and W. Ho, Phys. Rev. Lett. 93, 236802 (2004).

${ }^{23}$ Z. Sun, I. Swart, C. Delerue, D. Vanmaekelbergh, and P. Liljeroth, Phys. Rev. Lett. 102, 196401 (2009).

${ }^{24}$ G. Allan, C. Delerue, and Y. M. Niquet, Phys. Rev. B 63, 205301 (2001). 